\title{
A Selective Mnemonic Role for the Hippocampus in Monkeys: Memory for the Location of Objects
}

\author{
J. K. Parkinson, E. A. Murray, and M. Mishkin \\ Laboratory of Neuropsychology, National Institute of Mental Health, Bethesda, Maryland 20892
}

\begin{abstract}
Monkeys were trained preoperatively on a one-trial learning task in which they were required to associate in memory a novel object and the place in which it had just appeared. After learning the task to a level of $80 \%$ correct responses, they received bilateral ablations of either the hippocampal formation or the amygdaloid complex. The monkeys with amygdalectomy showed a small drop in performance initially but then regained their preoperative level. By contrast, the monkeys with hippocampectomy dropped to near-chance levels of performance and remained there throughout postoperative testing. Both groups performed at better than $90 \%$ correct responses on a test of recognition memory. These results, taken together with earlier work, suggest that although the hippocampus and amygdala appear to participate equally in object recognition, only the hippocampus is critical for the rapid formation of object-place associations.
\end{abstract}

Although the amygdaloid complex and hippocampal formation in monkeys appear to participate equally in object-recognition memory (Mishkin, 1978; Saunders et al., 1984; Murray and Mishkin, 1986), the amygdala has been found to be more important than the hippocampus for other types of object memory, such as object-punishment association (Weiskrantz, 1956), object-reward association (Spiegler and Mishkin, 1981; Phillips et al., 1983), and object-object association across modalities (Murray and Mishkin, 1985). Since the amygdala has thus been selectively implicated in some forms of object-associative memory, we wondered whether certain other forms might depend selectively on the hippocampus. The role of the 2 temporal lobe limbic structures together could then be viewed broadly as that of providing objects with associative meaning. If the hippocampus, like the amygdala, does indeed contribute selectively to certain forms of object-associative memory, then one of its roles, based on current evidence, could be that of associating objects with places. This idea derives from the finding that spatial reversal learning and spatial alternation in monkeys is impaired far more by hippocampectomy than by amygdalectomy (Waxler and Rosvold, 1970; Mahut, 1971; Jones and Mishkin, 1972); and it is further supported by an extensive literature on rodents suggesting that various forms of spatial memory in these animals are likewise critically dependent on the hippocampal system

\footnotetext{
Received Nov. 20, 1987; revised Mar. 18, 1988; accepted Apr. 5, 1988.

We thank Leon Dorsey and Linton Stokes for their valuable assistance.

Correspondence should be addressed to Mortimer Mishkin, Laboratory of Neuropsychology, National Institute of Mental Health, Building 9, Room 1N107, Bethesda, MD 20892.

Copyright (c) 1988 Society for Neuroscience $0270-6474 / 88 / 114159-09 \$ 02.00 / 0$
}

(O'Keefe et al., 1975; Olton and Samuelson, 1976; Becker et al., 1980; Morris et al., 1982; Thomas and Spafford, 1984).

To test the possibility that the hippocampus is essential for object-place association, monkeys were traincd to remcmbcr, on the basis of a single trial, the locations of 2 different objects. Their memory was assessed after a short delay with both "object-place" trials, in which the memory of the association was critical, and "place" trials, in which memory for location alone was sufficient. After mastering the task, the monkeys received ablations of either the hippocampal formation or the amygdaloid complex and were then retested. If the hippocampus is indeed selectively responsible for associating in memory the locations occupied by specific objects, then monkeys with hippocampectomy should be selectively impaired on the objectplace trials, while performing the place trials normally, whereas monkeys with amygdalectomy should perform normally on both types of trials. Alternatively, the hippocampal formation could play a more general role in the memory of spatial information, in which case the hippocampectomized monkeys might well be impaired on both trial types.

The monkeys were also trained postoperatively on a one-trial object-recognition task with short delays (delayed matching-tosample with trial-unique objects), in order to determine whether the experimental ablations produced any special difficulty with immediate memory for objects per se. Lack of an impairment on this task after hippocampectomy, combined with an impairment on the 1-trial object-place association task, would provide strong evidence for the selective involvement of this structure in some form of spatial memory.

A preliminary report of this work has appeared elsewhere (Parkinson and Mishkin, 1982).

\section{Materials and Methods}

\section{Subjects}

The subjects were 8 naive cynomolgus monkeys (Macaca fascicularis) weighing $2.5-4.0 \mathrm{~kg}$ at the beginning of the experiment. They were maintained on a diet of monkey chow supplemented with fruit, and water was always available.

\section{Apparatus and materials}

The monkeys were trained in a Wisconsin General Testing Apparatus (WGTA) inside a darkened room lined with sound-attenuating tiles. During test sessions, the test compartment of the WGTA was lit and the animal compartment was dark. Extrancous sounds were masked by white noise. The stimulus tray, which was stationary and at the level of the cage floor, contained 3 foodwells aligned $16 \mathrm{~cm}$ in front of the cage bars and spaced $16 \mathrm{~cm}$ apart. The stimuli consisted of 180 different objects that varied widely in color, shape, size, and texture. For each object, an exact duplicate was available. The objects were stored in 6 boxes, each of which contained 30 objects together with their duplicates. Different numbers of objects were required at different stages, with the 
Table 1. Schematization of the 12 trial configurations used in stages 3 and 4

\begin{tabular}{|c|c|c|c|c|c|c|c|}
\hline \multirow[b]{2}{*}{ Position: } & \multicolumn{3}{|c|}{ Acquisition setting } & \multicolumn{3}{|c|}{ Test setting } & \multirow{2}{*}{$\begin{array}{l}\text { Position } \\
\text { of correct } \\
\text { choice }\end{array}$} \\
\hline & 1 & 2 & 3 & 1 & 2 & 3 & \\
\hline & A & - & B & A & - & A & 1 \\
\hline & A & - & B & $\mathrm{B}$ & - & $\mathrm{B}$ & 3 \\
\hline & A & - & B & A & A & - & 1 \\
\hline & A & - & B & - & B & B & 3 \\
\hline & A & $\mathbf{B}$ & - & A & A & - & 1 \\
\hline & A & B & - & B & B & - & 2 \\
\hline & A & B & - & A & - & A & 1 \\
\hline & A & B & - & - & B & B & 2 \\
\hline & - & A & B & - & A & A & 2 \\
\hline & - & A & B & - & B & $\mathbf{B}$ & 3 \\
\hline & - & A & B & A & A & - & 2 \\
\hline & - & A & B & B & - & B & 3 \\
\hline
\end{tabular}

Capital letters $A$ and $B$ represent objects that are placed over the foodwells. (Note that in practice different pairs of objects are used on each trial.) The positions 1 , 2 , and 3 refer to the left, center, and right foodwells of the test tray, respectively. Each block of 4 trials comprises 1 of the 3 subtests of stage 3 . All 12 trial configurations were intermixed in a balanced order in stage 4 . The first 2 trials in each block of 4 represent the object-place trials and the second 2 in each represent the place trials.

final stage requiring the use of 48 different objects per session. This last requirement was fulfilled by using 2 boxes per session, with half the objects drawn randomly from each box; since the boxes were used in sequence, at least $2 \mathrm{~d}$ intervened between re-presentations of a given object.

\section{Preoperative training procedures \\ One-trial object-place association}

Stage 1 . The monkeys were first trained on a variation of delayed matching-to-sample in which both a trial-unique object and its position served as redundant memory cues. This stage consisted of 3 subtests administered in sequence. In the first subtest, only the 2 outermost foodwells were used. Each trial was composed of 2 parts, an acquisition phase and a choice test. For acquisition, the monkey was required to displace an unbaited sample object that was placed over either the left foodwell or the right in a pseudorandom sequence. Following a 6-sec delay, the monkey was given a choice test in which the sample, covering the same foodwell as before, was paired with an unlike object covering the other outermost foodwell. The monkey found a peanut half if it displaced the sample. After a 10-sec intertrial interval, the procedure was repeated with another pair of objects, and so on. On reaching criterion, the monkey was transferred to the second subtest, in which the 2 foodwells on the monkey's right were used. The testing procedures otherwise remained the same. Finally, on the third subtest, the 2 foodwells on the monkey's left were employed. The criterion for each subtest was set at $90 \%$ correct responses for each of 2 consecutive days. Animals were tested for 30 trials per day, $5 \mathrm{~d}$ per week. A noncorrection procedure was used, except in a few instances in which monkeys developed and maintained a position habit, and then correction training was introduced, but only briefly until the habit was broken. The correction procedure in these cases consisted of repeating the entire trial until the monkey made the correct choice. The position of the sample object was counterbalanced across trials with the constraint that the sample did not remain on the same side more than 3 times in succession.

Stage 2. The procedure was similar to that employed in stage 1 except that now the sample object appeared in a pseudorandom order over any one of the 3 foodwells and the 2 objects used for the choice test were identical (i.e., the sample object plus its duplicate). On the choice test, one object was presented in the same location in which it had appeared in the sample presentation, and the other was presented in 1 of the 2 remaining locations according to a predetermined pseudorandom schedule. Since the objects for choice were identical, only the location of the sample object could serve as a memory cue. Other testing procedures were identical to those in stage 1 .
Stage 3. This stage combined, within a single trial, some of the same procedures used in stages 1 and 2 . As in stage 1 , each subtest involved a different pair of the 3 foodwells. Now, however, an unlike pair of unbaited objects was presented in acquisition (say, $A$ on foodwell 1 and $B$ on foodwell 3 ), and the monkey was required to displace both. Then, after the 6-sec delay, 1 of the 2 sample objects (say A) together with its exact duplicate (a second $A$ ) were presented either over the same 2 foodwells that were used in acquisition ( $\mathrm{A}+$ on 1 and $\mathrm{A}-$ on 3 ) or over 1 of the 2 foodwells used for acquisition and over the third, previously unused, foodwell (A+ on 1 and A- on 2). Thus, half the trials (the first type just described) were object-place trials, which the animals could solve only by remembering the conjunction of each sample object and its location, whereas the other half (the second type) were place trials, which they could solve simply by remembering the locations of the sample objects (or, alternatively, the location of the uncovered well in acquisition and then avoiding it on the test). This procedure yielded 4 trial configurations per subtest, as illustrated in Table 1 . Testing continued for 24 trials per day (6 each of the 4 trial configurations per subtest), $5 \mathrm{~d}$ per week, until criterion was reached for each of the 3 subtests in succession. The intertrial interval was increased from 10 to $15 \mathrm{sec}$, and a noncorrection procedure was employed.

Stage 4. Trials on the 3 subtests of stage 3 were now intermixed in a balanced pseudorandom order. Two each of the 12 trial configurations used across subtests in stage 3 were combined within single test sessions in this final stage (see Table 1). Otherwise, the testing procedure was identical to that employed in stage 3 , with the trials equally divided between object-place and place trials. Since the acquisition phases for these 2 types of trials were identical, the monkeys had no cue as to which type was in use until the choice test. Testing continued for $25 \mathrm{~d}$. Scores from this stage were used to establish the preoperative baseline of performance.

\section{Surgery and histology}

The monkeys were divided into 2 groups matched for performance on the final design (stage 4). One-stage bilateral ablations were made aseptically under visual control through an operating microscope while the animals were under Nembutal anesthesia $(35 \mathrm{mg} / \mathrm{kg}$ ). For the hippocampectomy, the occipitotemporal convexity was elevated slightly with a brain spoon, and the tissue medial to the rostral half of the occipitotemporal sulcus was ablated with an aspirator until the ventricle was entered; the entire hippocampal formation and parahippocampal gyrus were then ablated, together with approximately the posterior half of the entorhinal cortex. The ventricular surface of the overlying portions of the temporal lobe served as a readily identifiable dorsolateral boundary for the removal, and the caudal, ventricular surface of the amygdala served as the rostral boundary. For the amygdalectomy, the frontotemporal junction was elevated slightly with a brain spoon, and the tissue medial to the anterior tip of the rhinal sulcus was entered with an aspirator; all gray matter rostral to the hippocampal formation and medial to the white matter of the temporal stem was then ablated. This ablation included the entire amygdaloid complex, the periamygdaloid cortex, and approximately the anterior half of the entorhinal cortex. Following surgery, the wound was closed in anatomical layers with silk sutures. Bicillin was administered intramuscularly as a prophylactic measure.

On completion of the experiment, the animals were given a lethal dose of anesthetic and perfused through the heart with saline and $10 \%$ buffered formalin solutions. The brains were removed, photographed, embedded in celloidin, and sectioned at $25 \mu \mathrm{m}$ in the frontal plane. Every 20 th section was stained with thionin and used to reconstruct the lesion.

The ablations in 3 of the monkeys in each group were essentially as intended. Histological reconstructions and representative sections through the lesions for these cases are shown in Figures 1 and 2. As illustrated in Figure 1, except for sparing of the posterior $2 \mathrm{~mm}$ of the left hippocampus in case $\mathrm{H} 1$, the hippocampal removals were complete. The only unintended damage evident in these animals was to a small portion of the caudal inferior temporal cortex bilaterally in case $\mathrm{H} 1$ and unilaterally in case $\mathrm{H} 3$.

Figure 2 shows the histological reconstruction for the cases with amygdalar ablations, which were also complete, although there was a variable amount of inadvertent damage in all 3 cases. Cases Al and A2 had bilateral damage to the tail of the caudate nucleus that extended rostrocaudally for $2 \mathrm{~mm}$ in each animal on the right and for 8 and $4 \mathrm{~mm}$, respectively, on the left. Case $\mathrm{A} 3$ had only a small amount of damage to the tail of the caudate nucleus on the right, extending rostrocaudally 

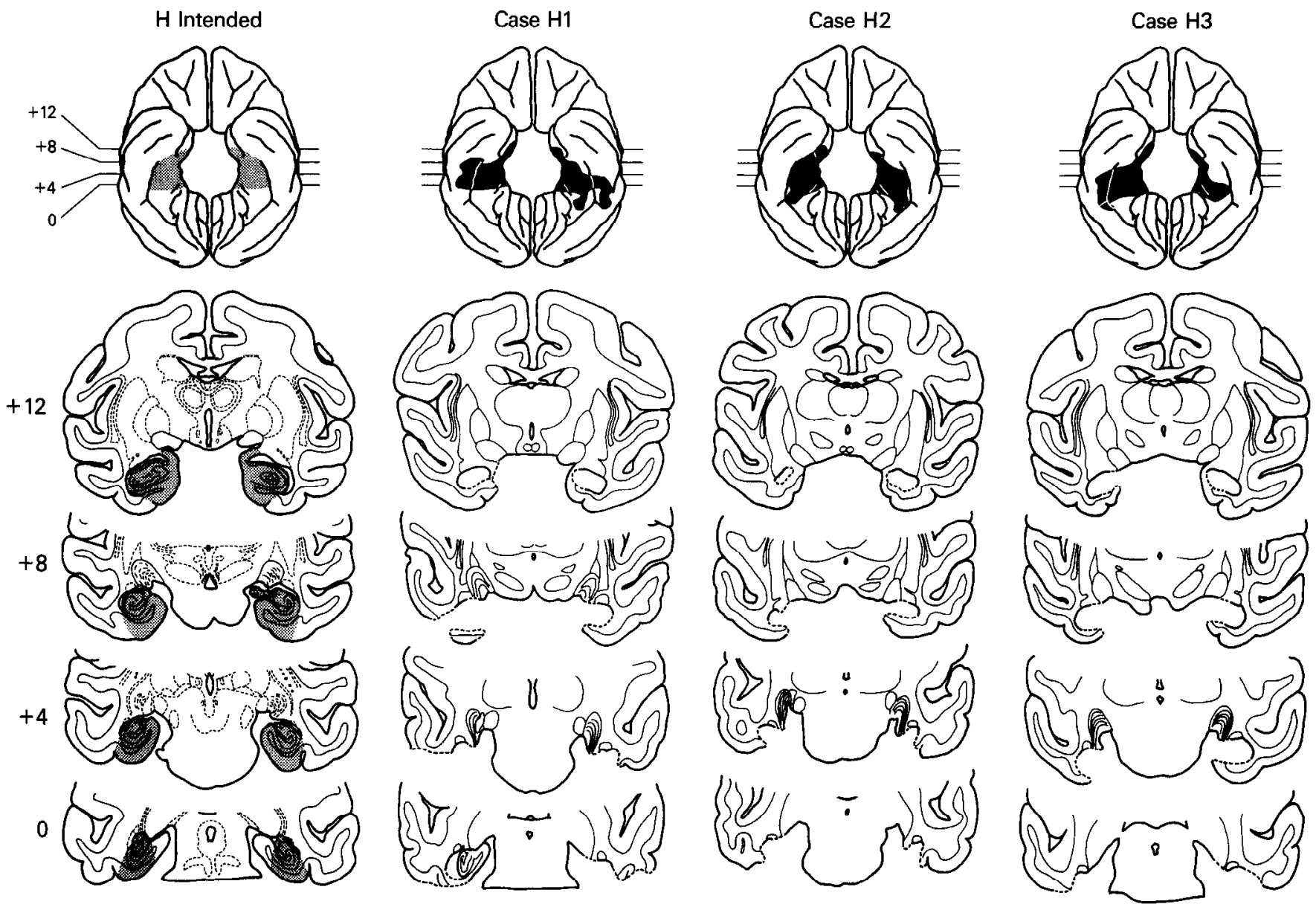

Figure 1. Surface reconstructions of the lesions on a standard ventral view of a macaque brain (top) and coronal sections through the lesions (below) for 3 monkeys with hippocampal ablations (cases $\mathrm{H} 1-\mathrm{H} 3$ ). The intended lesion (left column) is indicated by the stippled area on the ventral view and on the coronal sections of a normal brain. Actual lesions in cases $\mathrm{H} 1-\mathrm{H} 3$ are shown in black on the ventral views and by dashed lines in the coronal sections. Note inadvertent damage to caudal inferior temporal cortex bilaterally in case $\mathrm{Hl}($ levels $+8,+4,0)$ and unilaterally in case $\mathrm{H} 3$ (levels $+4,0$ ). For ease in visual matching of cross sections to the ventral reconstructions, the ventral view has been reversed (i.e., the left hemisphere is shown at left) in this and in the following 2 figures. Numerals indicate distance (in $\mathrm{mm}$ ) from the interaural plane (0).

for approximately $0.5 \mathrm{~mm}$. This monkey also sustained bilateral damage to the ventral putamen for a rostrocaudal distance of $2.5 \mathrm{~mm}$ on the left and $1.0 \mathrm{~mm}$ on the right.

The 2 remaining monkeys (one in each group) sustained significant inadvertent damage to structures known to be important for vision. The histological reconstructions for these 2 monkeys are shown in Figure 3. Like the other amygdalectomized subjects, case A4 received a complete ablation of the amygdala plus some damage to the tail of the caudate nucleus. Unlike the others, however, case A4 also sustained bilateral cell loss and gliosis in the dorsal lateral geniculate nucleus and lateral pulvinar. This bilateral neuronal degeneration, which was particularly severe on the left, resulted from bilateral infarctions located in the white matter immediately lateral to the caudal portion of the pulvinar and involving the optic radiations (see Fig. 3, level 0).

Like the other hippocampectomized monkeys, case $\mathrm{H} 4$ received a complete hippocampal removal, but, as a result of infarction, this animal also sustained a substantial amount of damage to the rostral inferior temporal cortex on the left. In addition, moderate cell loss and gliosis were evident in the dorsal lateral geniculate nucleus and lateral pulvinar on the right. This was due to the same type of optic radiation infarction described above for case A4.

Because of the unintended bilateral damage to the visual system in these 2 monkeys (at the level of the thalamus in A4, and at the level of the thalamus on one side and the inferior temporal cortex on the other side in $\mathrm{H} 4)$, their data were considered separately from those of the others.

\section{Postoperative training procedures}

One-trial object-place association. Following 10-14 d of postsurgical recovery, the monkeys were retested on the final stage (stage 4). Testing continued at the rate of 24 trials per session until the monkey surpassed its own preoperative mean score for 2 consecutive 5-d blocks of 120 trials each or for a maximum of $75 \mathrm{~d}$ (1800 trials).

Delayed matching-to-sample. Following postoperative testing on stage 4 , the monkeys were trained on a form of delayed matching-to-sample (DMS). The testing was conducted in a manner similar to that used previously by Parkinson and Medin (1983) to investigate whether familiarity and novelty are distinct discriminative attributes in memory. Highly familiar objects (F) were mixed with novel objects $(\mathrm{N})$ within trials to yield 4 different trial types $(\mathrm{F}+\mathrm{N}-, \mathrm{N}+\mathrm{N}-, \mathrm{N}+\mathrm{F}-$, and $\mathrm{F}+\mathrm{F}-$ ) that were counterbalanced within single test sessions. For present purposes, data analysis was limited to the $\mathrm{N}+\mathrm{N}-$ - trial type, which corresponds to DMS with trial-unique objects. A set of approximately 1300 objects was available for the $10 \mathrm{~d}$ of testing, so no object was used more than once. Each trial was composed of 2 parts, sample presentation and choice test. On the sample presentation, the monkey displaced the sample object overlying the unbaited central well of a 3-well test tray. On the choice test, $10 \mathrm{sec}$ later, the sample and comparison objects appeared over the lateral wells of the test tray, and now the monkey could obtain a half-peanut reward by displacing the sample. The monkeys were trained with $15-\mathrm{sec}$ intertrial intervals at a rate of 32 trials per day, consisting of 8 each of the 4 different trial types. 

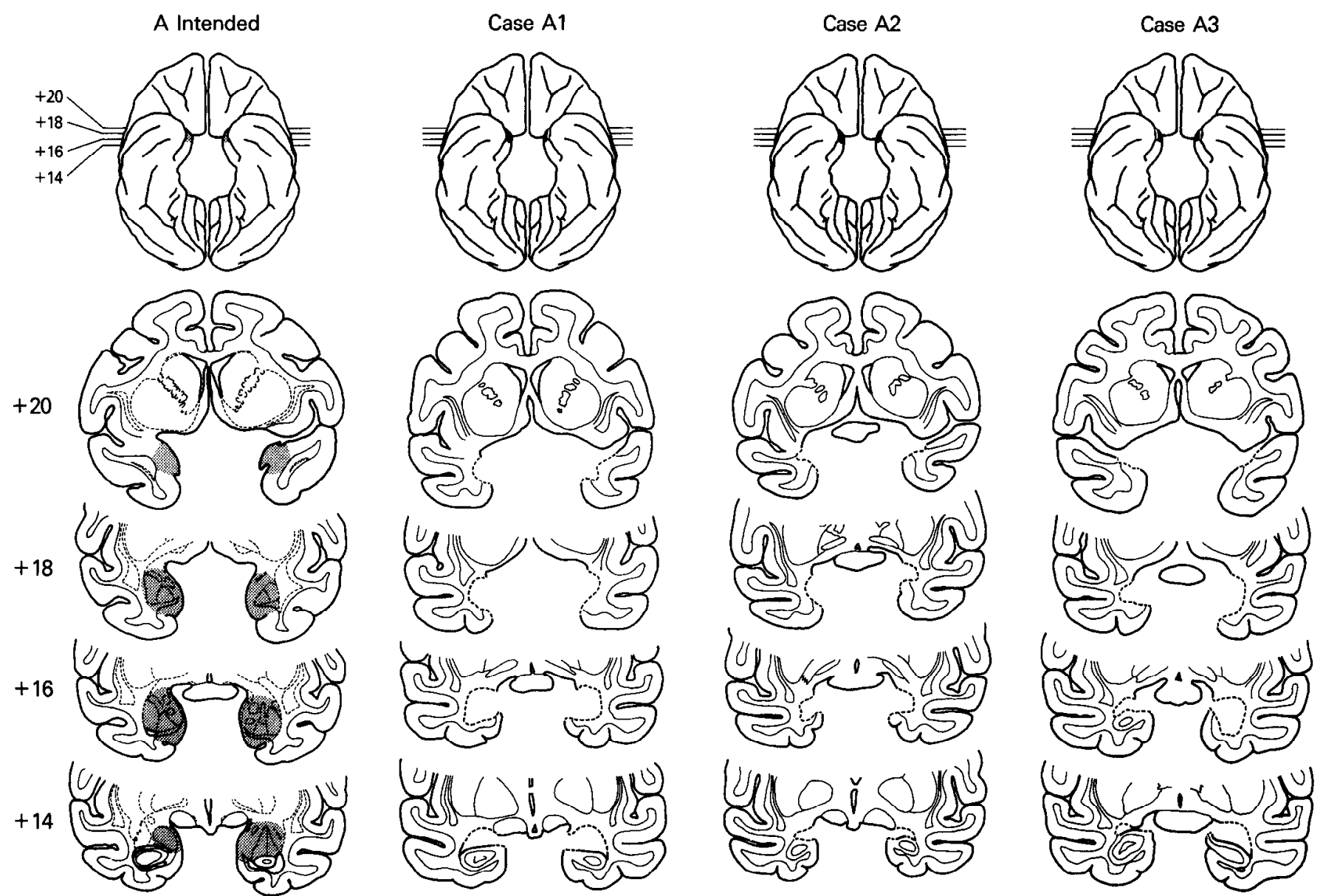

Figure 2. Ventral surface reconstructions of the lesions (top) and coronal sections through the lesions (below) in 3 monkeys with amygdalar ablations (cases Al-A3). Intended removal, left column. Conventions as in Figure 1.

\section{Results}

One-trial object-place association

Summary. The major results are illustrated in Figure 4. Preoperatively, the monkeys averaged slightly over $80 \%$ correct responses on the final stage of the one-trial object-place association task. Following surgery, the monkeys with amygdalectomy (cases A1-A3) showed a small drop in performance initially but then regained their preoperative level of performance. By contrast, the monkeys with hippocampectomy (cases H1$\mathrm{H} 3$ ) dropped to near-chance levels of performance and remained there for the entire period of postoperative training. A detailed description of the results follows.

Preoperative learning and performance. Preoperatively, the monkeys required an average of 1694 trials and 482 errors to attain criterion (stage 1, 727 trials and 255 errors; stage 2, 420 trials and 102 errors; stage 3,547 trials and 125 errors). On stage 4 , in which the trial configurations of the 3 subtests of stage 3 were intermixed for a 25 -d performance test, the animals averaged $81.5 \%$ correct responses. The groups scheduled to receive hippocampectomy and amygdalectomy scored 81.4 and $81.6 \%$ correct responses, respectively, and they remained well matched when the 2 animals with the unintended damage to the visual system were excluded (H1-H3, 81.5\%; A1-A3, 80.8\%). As shown in Figure 5, the scores on place trials were higher than those on object-place trials for both groups, and this was re- flected as a significant main effect of trial type in the ANOVA with repeated measures $[F(1,4)=93.74, p<0.01]$. No other effects were significant.

Postoperative performance. Postoperative testing was divided into 3 segments of $25 \mathrm{~d}$ (600 trials) each, but only the first 2 segments were analyzed since some monkeys were not tested on the third. In the first segment, both the group factor and trial type were significant $[F(1,4)=41.23, p<0.01$, and $F(1,4)=$ $13.29, p<0.05$, respectively], and there was no interaction between them (see Fig. 5). That is, just as they had preoperatively, all the monkeys performed slightly better on the place trials than on the object-place trials, and, as a result (see Table 2 ), the hippocampectomized monkeys were equally impaired on both trial types. In the second segment of testing, the group factor remained significant $[F(1,4)=57.06, p<0.01]$, but the effect of trial type fell short of significance. Again, there was no interaction between the 2 factors.

Case A4, excluded from the amygdalectomized group becausc of the inadvertent bilateral damage to the visual system, never regained its preoperative level of performance $(84.2 \%$ correct responses). For the first 5-d block following surgery, this monkey averaged only $47 \%$ correct responses. By the eighth 5 -d block, however, it achieved $77.5 \%$ correct responses, a level that it was able to maintain but not exceed for the remainder of testing. Presumably, this animal sustained visual field defects that prevented visual discrimination during early postoperative train- 


\section{Case A4}
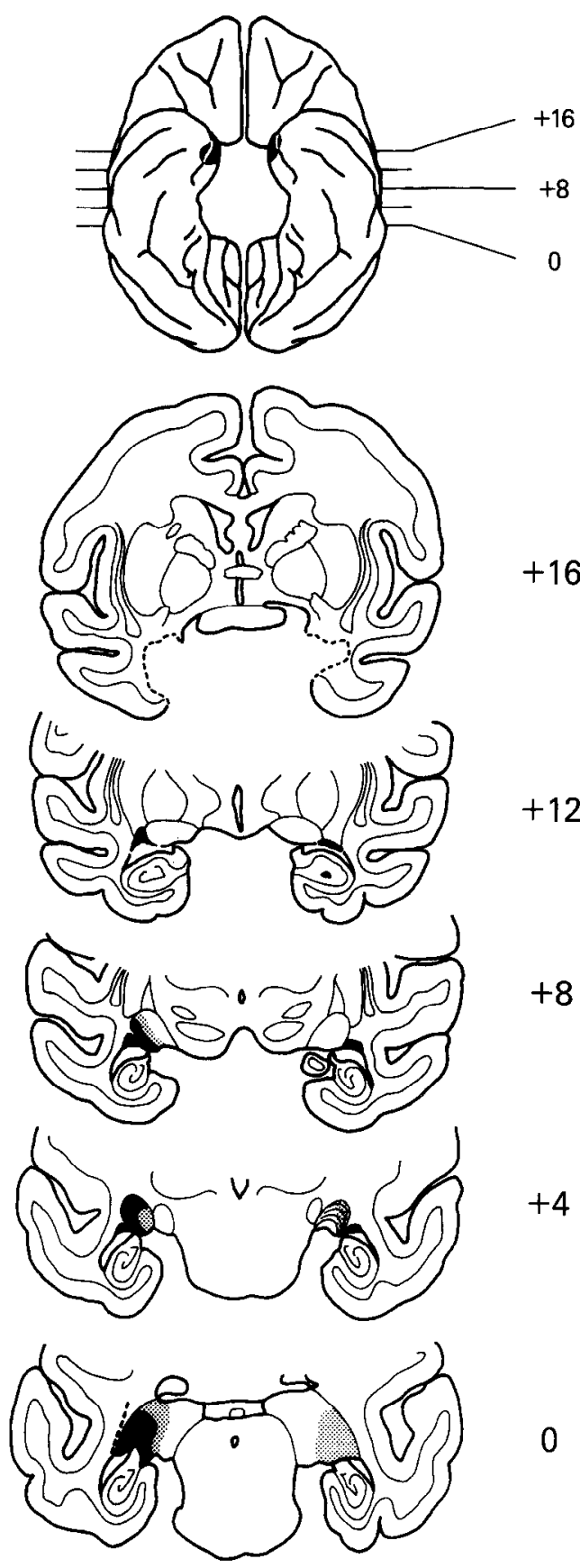

Case $\mathrm{H} 4$

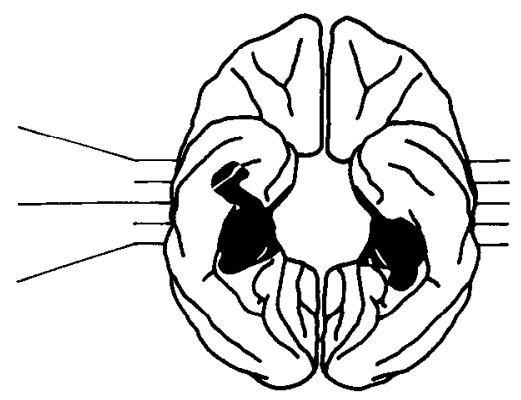

$+16$

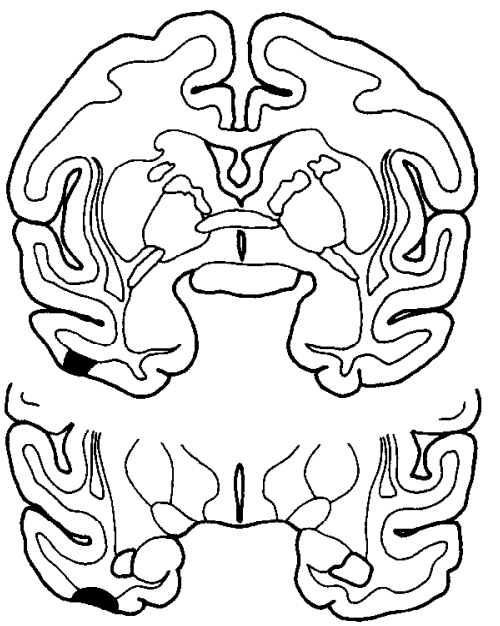

$+8$

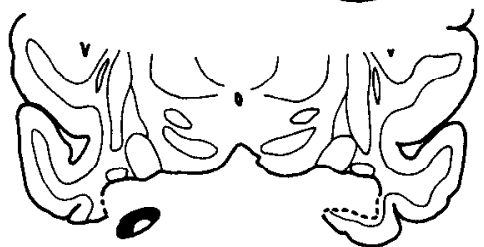

$+4$

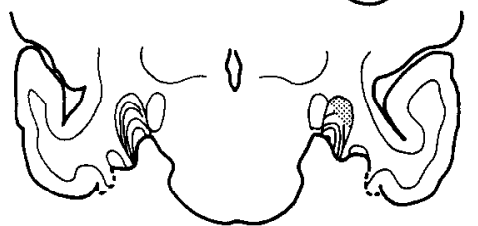

0

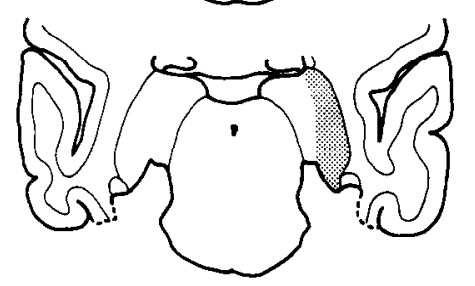

Figure 3. Ventral surface reconstructions and coronal sections through the lesions for one monkey with an amygdalectomy (case A4) and one monkey with a hippocampectomy (case $\mathrm{H} 4$ ). Regions of complete cell loss are shown in black, and regions of partial cell loss and gliosis by stippling. Other conventions as in Figure 1. Case A4 sustained bilateral cell loss in the dorsal lateral geniculate nucleus and pulvinar, more extensive on the left than on the right (levels $+8,+4,0$ ), as well as bilateral damage to the tail of the caudate nucleus (levels $+12,+8,+4)$. Case $\mathrm{H} 4$ sustained damage to the rostral inferior temporal cortex on the left (levels +16 , +12 ) and to the dorsal lateral geniculate nucleus and pulvinar on the right (levels 14,0 ). All of these unintended lesions resulted from infarctions.

ing, but it was later able to overcome this handicap, at least partially. [The finding in case A4, which was, in fact, the first case in its group to be tested, together with the initial postoperative results from the second animal in the group, formed the basis of an erroneous preliminary report (Mishkin et al., 1982) that amygdalectomy as well as hippocampectomy impaired memory for object-place association.] Case H4, also excluded from its group because of bilateral damage to the visual system, performed as poorly as the other hippocampectomized monkeys (an average of $53.2 \%$ correct responses for its entire postoperative course, as compared with an average of $57.2 \%$ for the others). Although a detrimental effect of the extra damage was not detectable on this task, such an effect did appear on the next task.

\section{Delayed matching-to-sample}

Performance on DMS (trial type $\mathrm{N}+\mathrm{N}-$, see postoperative training procedures) averaged $94.2 \%$ correct responses for the amygdalectomized monkeys and $91.2 \%$ for the hippocampectomized monkeys (see Fig. 6). The 2 groups did not differ significantly (Mann-Whitney $U$-test, $U=2, p=0.20$ ). Case A4 achieved $91.2 \%$ correct responses, a finding consistent with this animal's substantial recovery of the ability to perform accurately on the object-place task. Unlike the other animals in both groups, 
Figure 4. Performance on the 1-trial object-place association task. Mean scores for the amygdalectomized monkeys (cases A1-A3) and hippocampectomized monkeys $(\mathrm{H} 1-\mathrm{H} 3)$ are indicated by the open circles and filled circles, respectively. Each point represents a block of $5 \mathrm{~d}$ of testing. Vertical lines through each point indicate the range of scores for the group. From the 11 th through the 15 th blocks, only 2 hippocampectomized monkeys (H1 and H2) were tested.
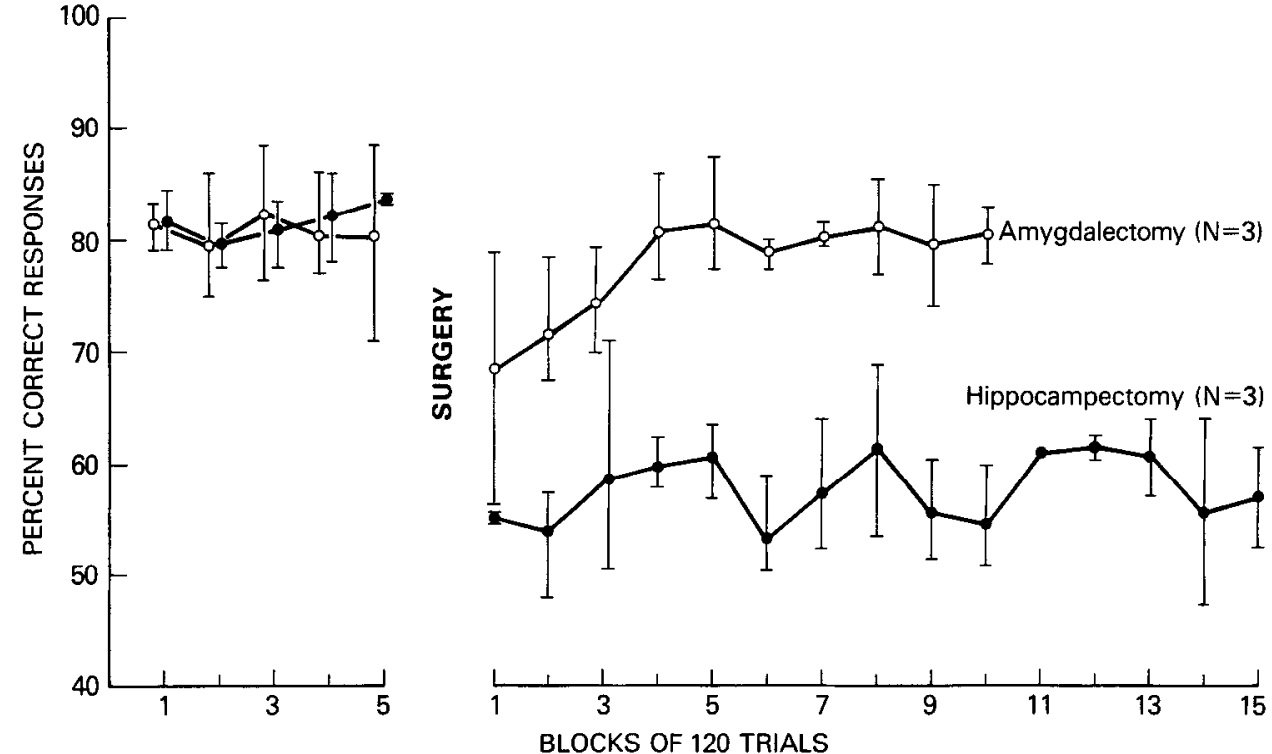

however, case $\mathrm{H} 4$ achieved only $66.2 \%$ correct responses. Presumably, the damage to the visual system that this monkey sustained, in contrast to the damage in case A4, led to a combination of visual field defect and higher-order perceptual loss that interfered with visual recognition of objects.

\section{Discussion}

The present results confirm earlier findings that neither hippocampectomy nor amygdalectomy affects a monkey's immediate memory for object quality per se (Mishkin, 1978; Murray and Mishkin, 1984, 1986). An impairment in such object-recognition ability after hippocampectomy becomes evident only when the memory requirement is increased beyond $10 \mathrm{sec}$ or so, and the impairment becomes truly severe only when the hippocampal removal is combined with removal of the amygdala (Mishkin, 1978; Murray and Mishkin, 1984, 1986). These earlier findings, considered together with the finding in the present study of an impairment after hippocampectomy alone in object-place association, are consistent with the view that the hippocampus participates in at least 2 memory processes. First, in concert with the amygdala, the hippocampus plays a critical role in object recognition beyond the immediate memory span, although, as just indicated, this critical role does not become evident unless both structures are removed in combination. Second, the hippocampus is critical by itself for ccrtain associative processes, even in immediate memory, and in this domain its role is complementary to that of the amygdala. This point is elaborated below.

Considered alone, the data from the present experiment might allow the proposal that the hippocampus is necessary for associating in memory 2 or more stimulus qualities or stimulus events, whatever the perceptual modalities involved. As pointed out in the introduction, however, the previously accumulated evidence does not permit any such conclusion. To the contrary, monkeys with hippocampal-system lesions alone are unimpaired on a variety of associative memory tests, many of which present severe difficulty instead for monkeys with amygdalectomy. Examples include: immediate memory for object-reward association, a task that requires remembering on the basis of a single trial whether or not an object had just been baited with
Figure 5. Comparison of performance on the 2 trial types in the 1-trial object-place association task. The monkeys consistently scored higher on the place trials than on the object-place trials, and this difference in performance on the 2 trial types was significant for the 5 blocks of trials preoperatively $(P R E-O P)$, as well as for the first 5 blocks of trials postoperatively (POST-OP I) but not for the second 5 blocks of trials postoperatively (POST $O P I I$.
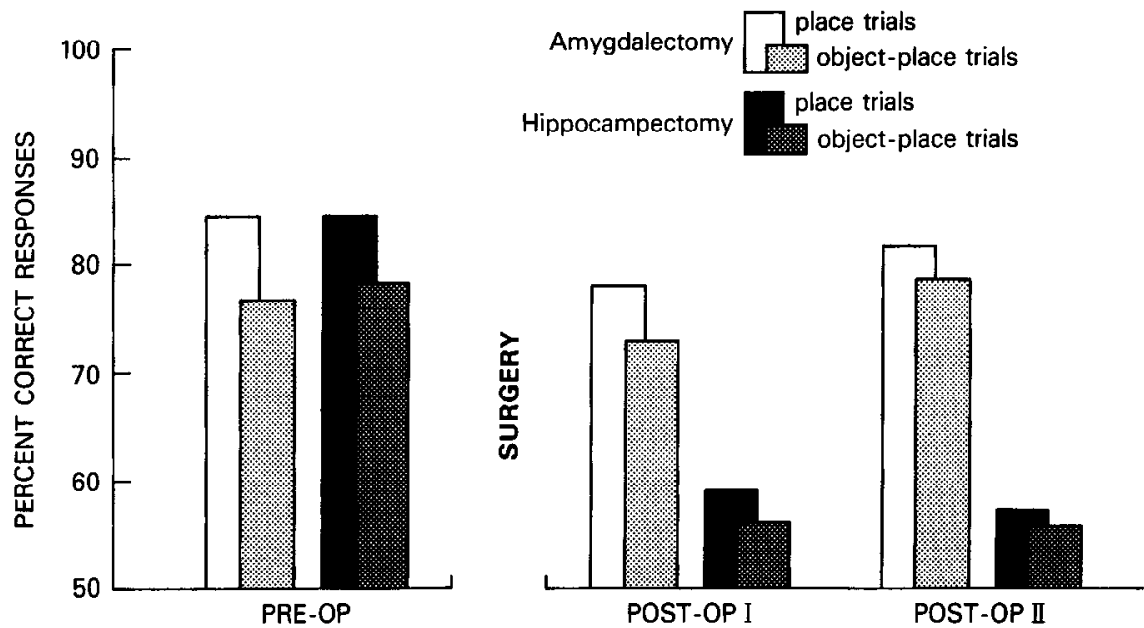
Table 2. Average percentage of correct responses for each monkey across 25 test sessions ( 300 trials for each trial type per 25 sessions) administered either preoperatively (Pre-op) or postoperatively (Postop)

\begin{tabular}{|c|c|c|c|c|c|c|c|c|}
\hline \multirow[b]{3}{*}{ Animal } & \multirow{2}{*}{\multicolumn{2}{|c|}{$\begin{array}{l}\text { Pre-op } \\
\text { sessions } \\
1-25\end{array}$}} & \multicolumn{6}{|c|}{ Post-op sessions } \\
\hline & & & \multicolumn{2}{|c|}{$1-25$} & \multicolumn{2}{|c|}{$26-50$} & \multicolumn{2}{|c|}{$51-75$} \\
\hline & $\overline{\mathrm{O}-\mathrm{P}}$ & $\mathbf{P}$ & $\overline{\mathrm{O}-\mathrm{P}}$ & $\mathrm{P}$ & $\overline{\mathrm{O}-\mathrm{P}}$ & $P$ & $\mathrm{O}-\mathrm{P}$ & $\mathrm{P}$ \\
\hline $\mathrm{H} 1$ & 76 & 83 & 53 & 58 & 56 & 53 & 55 & 57 \\
\hline $\mathrm{H} 2$ & 79 & 86 & 55 & 57 & 50 & 55 & - & - \\
\hline $\mathrm{H} 3$ & 80 & 85 & 60 & 63 & 62 & 63 & 62 & 62 \\
\hline $\bar{X}(n=3)$ & 78 & 85 & 56 & 59 & 56 & 57 & - & - \\
\hline $\mathrm{H} 4$ & 78 & 84 & 52 & 54 & 55 & 53 & 53 & 52 \\
\hline Al & 76 & 87 & 73 & 80 & 78 & 84 & 81 & 80 \\
\hline A2 & 73 & 79 & 68 & 76 & 82 & 80 & - & - \\
\hline A3 & 82 & 88 & 78 & 79 & 77 & 81 & - & - \\
\hline $\bar{X}(n=3)$ & 77 & 85 & 73 & 78 & 79 & 82 & - & - \\
\hline A4 & 82 & 86 & 49 & 50 & 63 & 68 & 70 & 78 \\
\hline
\end{tabular}

Abbreviations: $\mathrm{H}$, bilateral hippocampectomy; A, bilateral amygdalectomy; $\mathrm{O}$ $P$, object-place trials; $P$, place trials.

food reward (Spiegler and Mishkin, 1981; Phillips et al., 1983); discrimination learning with secondary reinforcement, tasks requiring the mnemonic association of neutral auditory or visual stimuli with food reward (Gaffan and Harrison, 1987; D. Gaffan, personal communication); and cross modal (tactual-to-visual) delayed nonmatching-to-sample (Murray and Mishkin, 1985), a task requiring the conjunction, in memory, of the tactual and visual qualities of individual objects. Finally, we have recently found that monkeys with hippocampal ablations are unimpaired in either the retention or new learning of arbitrary associations between pairs of visual stimuli, i.e., memory for visual-visual associations (Murray et al., 1988). It should be noted that the associative memory tasks just cited (except for those involving secondary reinforcement) are similar to the one in the present study in that they too involved presentation of sample stimuli followed shortly thereafter by the presentation of choice stimuli. The major difference is that in the present study the sensorysensory conjunction was between an object and its spatial location, and in this case, contrary to the others, it is clear that amygdalectomy had little effect, whereas hippocampectomy nearly abolished the ability. This discovery of a selective mnemonic role for the hippocampus, combined with the earlier evidence of a selective mnemonic role for the amygdala (Spiegler and Mishkin, 1981; Murray and Mishkin, 1985), supports the proposal advanced in the introduction that these 2 limbic structures provide objects with complementary associative contexts or meanings.

Although the object location memory of the animals with hippocampal lesions was essentially eliminated, it is not yet clear that this was their fundamental impairment. Because hippocampectomized monkeys perform well on spatial delayed response with short delays (Mishkin, 1954; Correll and Scoville, 1967; Zola-Morgan and Squire, 1985; Murray and Mishkin, 1986), it was considered likely that they would also perform well on the place trials in the present study. Clearly, however, the monkeys with hippocampal removals were as severely impaired on the place trials as they were on the object-place trials. There are at least 2 possible explanations for this finding. First, unlike spatial delayed response, which involves direct cueing

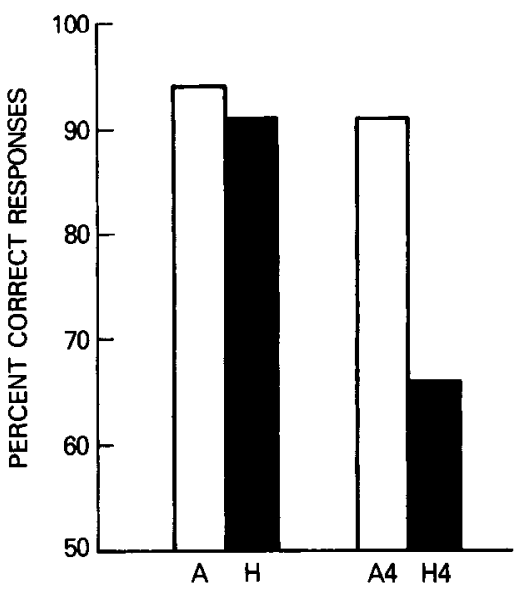

Figure 6. Performance on delayed matching-to-sample. $A$, Mean scores for cases A1-A3; $H$, mean scores for cases $\mathrm{H} 1-\mathrm{H} 3 ; A 4$ and $H 4$ scores of the 2 monkeys with inadvertent bilateral damage to the visual system.

with food of 1 out of 2 wells, the place trials of the present study involved indirect cueing with objects of 2 out of 3 wells. The hippocampectomized monkeys may thus have been impaired in the present study because of the increased load on place memory or, alternatively, because a different mnemonic strategy is needed for dealing with 3 places than for dealing with 2 places. (Presumably, they did not adopt the strategy of simply remembering the uncued well for later avoidance, for then the place trials should have presented no greater challenge than spatial delayed response.) In any event, perhaps because they could not remember 2 places out of 3 , the monkeys were necessarily also unable to associate in memory a different object with each place. According to this first interpretation, then, the basic impairment after hippocampectomy is in remembering 2 or more places. A second possibility is just the reverse of the first; that is, the hippocampectomized monkeys may have failed on the place trials due to a basic impairment in remembcring object-place associations. Because the monkeys had no indication during the acquisition phase as to which of the 2 trial types was in force, and because remembering places alone was inadequate for performing the object-place trials, they may never have adopted a strategy of remembering places only. This interpretation is supported by the finding that all the monkeys performed only slightly (though significantly) better on the place trials than on the object-place trials, a small advantage that was not eliminated by hippocampectomy. Presumably, in preparation for either trial type, the monkeys attempted to remember all the information available at acquisition. Then, on the choice test, to avoid the incorrect place, the monkey had to recall either that this place, although now covered, had not been covered in acquisition (place trials) or that it had been covered, but with a different object (object-place trials). Clearly, the former type of trial is the simpler of the two, and this could easily account for the slight but consistent superiority on it exhibited by all animals.

Whichever memory impairment is the more fundamental, whether for place or for object-place association, the monkeys with hippocampal ablations were clearly incapable of associating objects and their spatial locations. A similar result was obtained recently by Gaffan and Saunders (1985), who found that monkeys with fornix transection were impaired on a run- 
ning recognition version of a stimulus-place association task. Both findings are consistent with those of Smith and Milner (1981), who reported that epileptic patients given right temporal lobe excisions that included a large portion of the hippocampal formation were severely impaired in reproducing the spatial locations of an array of objects they had viewed earlier. By contrast, patients with excisions on the left comparable to those on the right, or with excisions on the right that largely spared the hippocampal formation, performed at a level equal to that of normal control subjects. The present findings strongly support the conjecture by Smith and Milner (1981) that the affected group was impaired because of their right hippocampal damage, specifically, and not because of combined damage to the right amygdala. It is of interest, however, that, unlike the impairment in the monkeys with bilateral hippocampectomy, the impairment in the patients with extensive right hippocampal damage was evident only in a delayed, and not in an immediate, recall condition (Smith and Milner, 1988). This difference suggests that immcdiatc rccall of objcct location (unlikc that for most other material) might well be impaired in a patient that had sustained bilateral rather than unilateral hippocampal damage. In support of the differential susceptibility of object-location memory to hippocampal damage in humans, and in agreement with the results in monkeys, there was a delay condition at which recall of object location was impaired but memory of the objects themselves was not (Smith and Milner, 1981). Furthermore, the same patients that had abnormal difficulty in recalling the spatial locations of visual stimuli were found to have no such difficulty in recalling the frequencies of occurrence of visual stimuli (Smith and Milner, 1983). These results support the view that the memory loss for object location in patients with right hippocampal resections, like that in monkeys with bilateral hippocampectomy, is highly selective.

\section{Spatial interpretations of hippocampal function}

The present results are consistent with at least 2 current interpretations of hippocampal function, both of which place heavy emphasis on the role of the hippocampus in spatial memory. One, the cognitive map hypothesis of O'Keefe and Nadel (1978, 1979), holds that the hippocampus constructs and stores representations of the "spatial layout of an animal's experienced environment" and that these representations constitute the basis of a nonegocentric form of spatial memory. Numerous studies have now implicated the hippocampal formation in some form of maplike memory, both through the discovery and documentation of "place" cells in the rat hippocampus (O'Keefe and Dostrovsky, 1971; Ranck, 1973; O'Keefe, 1976, 1979) and the demonstration of severe impairments on maze learning in both rats and monkeys after hippocampal-system damage (O'Keefe et al., 1975; Olton and Samuelson, 1976; Becker et al., 1980; Morris et al., 1982; Murray et al., 1988). The second hypothesis with which the present results are consistent stresses the role of the hippocampus in learning the spatial relations of objects relative to the perceiver's body, as well as in learning spatially directed movements (Gaffan, 1988; Gaffan and Harrison, 1988). From this evidence, Gaffan has argued that damage to the hippocampal system may lead to an impairment in "personal memory." But although the present results are consistent with these relatively limited, spatial interpretations of hippocampal function, both the cognitive map and personal memory hypotheses have, in fact, been extended beyond the spatial domain to en- compass memory ability in a more general sense. Thus, the cognitive map hypothesis has been extended to encompass most, if not all, associative (contextual) memory abilities (O'Keefe and Nadel, 1978), and, similarly, the personal memory hypothesis has been generalized to include nonspatial forms of personal memory as well (Gaffan, 1988). In this way, these 2 interpretations have been used to suggest that damage to the hippocampal system alone can account for the human syndrome of global anterograde amnesia. As we have pointed out above and elsewhere (Mishkin, 1978, 1982; Murray and Mishkin, 1984, $1985,1986)$, the evidence from ablation studies in monkeys suggests that many important nonspatial forms of recognition and associative memory depend critically on the amygdalar system rather than, or in addition to, the hippocampal system and that, therefore, instances of truly profound and global anterograde amnesia are likely to involve combined damage to both systems.

\section{Neural substrates of object-place association}

Earlier, we (Murray and Mishkin, 1985) reported that the amygdala mediates at least one form of cross-modal associative memory and suggested that it is likely to mediate others as well. In speculating about the neural pathways underlying such crossmodal associative processes, we proposed that, since all the sensory systems project to the amygdala either directly or indirectly through a series of neocortical stations and since, in addition, the amygdala returns projections to all the cortical sensory systems, these corticoamygdalocortical pathways might allow the formation of connections between stored representations within different sensory systems. By extrapolation, the corticohippocampocortical pathways might allow the formation of connections between the stored representations of objects and the stored representations of the places that they occupy. Specifically, we suggest that the visual object recognition aspect of object-place association depends on a pathway from the inferior temporal cortex through the perirhinal and entorhinal cortices into the hippocampal formation, a pathway parallel to that projecting from the inferior temporal cortex into the amygdala that is presumed to underlie the association of recognized visual objects with the nonvisual qualities of those same objects (Murray and Mishkin, 1985), as well as with food rewards (Jones and Mishkin, 1972; Spiegler and Mishkin, 1981; Gaffan et al., 1988). Presumably, the mnemonic information about the locations of environmental stimuli relative to each other and to the perceiver's body is provided by the spatial sensory processing systems involving the occipitoparietoprefrontal pathway (Mishkin, 1972; Pohl, 1973), and this information reaches the presubicular, perirhinal, and entorhinal cortices either directly (Jones and Powell, 1970; Seltzer and Van Hoesen, 1979; Goldman-Rakic et al., 1984) or indirectly via the cingulate gyrus (Jones and Powell, 1970; Baleydier and Mauguiere, 1980; Pandya et al., 1981), the parahippocampal gyrus (Jones and Powell, 1970; Pandya et al., 1981; Goldman-Rakic et al., 1984), or both. Furthermore, as in the case of the amygdala, all of the projections into the hippocampus via the perirhinal and entorhinal cortices are reciprocated. Thus, just as the amygdala is in a position to bring together information about the quality of environmental stimuli arising from different sensory modalities, the hippocampus may be in a position to bring together information about environmental stimuli and their locations relative both to other stimuli and to the organism itself. 


\section{References}

Baleydier, C., and F. Mauguiere (1980) The duality of the cingulate gyrus in monkey. Neuroanatomical study and functional hypothesis. Brain 103: 525-554.

Becker, J. T., J. A. Walker, and D. S. Olton (1980) Neuroanatomical bases of spatial memory. Brain Res. 200: 307-320.

Correll, R. E., and W. B. Scoville (1967) Significance of delay in the performance of monkeys with medial temporal lobe resections. Exp. Brain Res. 4: 85-96.

Gaffan, D. (1988) Amnesia, personal memory and the hippocampus: Experimental neuropsychological studies in monkeys. In Cognitive Neurochemistry, S. M. Stahl, S. D. Iversen, and E. C. Goodman, eds. (in press).

Gaffan, D., and S. Harrison (1987) Amygdalectomy and disconnection in visual learning for auditory secondary reinforcement by monkeys. J. Neurosci. 7: 2285-2292.

Gaffan, D., and S. Harrison (1988) A comparison of the effects of fornix transection and sulcus principalis ablation upon spatial learning by monkeys: Personal space and conditional rules. Behav. Brain Res. (in press).

Gaffan, D., and R. C. Saunders (1985) Running recognition of configural stimuli by fornix-transected monkeys. Q. J. Exp. Psychol. 37B: 61-71.

Gaffan, E. A., D. Gaffan, and S. Harrison (1988) Disconnection of the amygdala from visual association cortex impairs visual reward-association learning in monkeys. J. Neurosci. 8: 3144-3150.

Goldman-Rakic, P. S., L. D. Selemon, and M. L. Schwartz (1984) Dual pathways connecting the dorsolateral prefrontal cortex with the hippocampal formation and parahippocampal cortex in the rhesus monkey. Neuroscience 12: 719-743.

Jones, B., and M. Mishkin (1972) Limbic lesions and the problem of stimulus reinforcement associations. Exp. Neurol. 36: 362-377.

Jones, E. G., and T. P. S. Powell (1970) An anatomical study of converging sensory pathways within the cerebral cortex of the monkey. Brain 93: 793-820.

Mahut, H. (1971) Spatial and object reversal learning in monkeys with partial temporal lobe ablations. Neuropsychologia 9: 409-424.

Mishkin, M. (1954) Visual discrimination performance following partial ablations of the temporal lobe: II. Ventral surface vs. hippocampus. J. Comp. Physiol. Psychol. 47: 187-193.

Mishkin, M. (1972) Cortical visual areas and their interactions. In Brain and Human Behavior, A. G. Karczmar and J. C. Eccles, eds., pp. 187-208, Springer-Verlag, Berlin.

Mishkin, M. (1978) Memory in monkeys severely impaired by combined but not by separate removal of amygdala and hippocampus. Nature 273: 297-298.

Mishkin, M. (1982) A memory system in the monkey. Phil. Trans. R. Soc. London 298: 85-95.

Mishkin, M., B. J. Spiegler, R. C. Saunders, and B. L. Malamut (1982) An animal model of global amnesia. In Alzheimer's Disease: $A$ Report of Progress, S. Corkin, ed., pp. 235-247, Raven, New York.

Morris, R. G. M., P. Garrud, J. N. P. Rawlins, and J. O'Keefe (1982) Place navigation impaired in rats with hippocampal lesions. Nature 297: 681-683.

Murray, E. A., and M. Mishkin (1984) Severe tactual as well as visual memory deficits follow combined removal of the amygdala and hippocampus in monkeys. J. Neurosci. 4: 2565-2580.

Murray, E. A., and M. Mishkin (1985) Amygdalectomy impairs crossmodal associations in monkeys. Science 228: 604-606.

Murray, E. A., and M. Mishkin (1986) Visual recognition in monkeys following rhinal cortical ablations combined with either amygdalectomy or hippocampectomy. J. Neurosci. 6: 1991-2003.

Murray, E. A., D. Gaffan, and M. Mishkin (1988) Role of the amygdala and hippocampus in visual-visual associative memory in rhesus monkeys. Soc. Neurosci. Abstr. 14:2.
Murray, E. A., M. Davidson, D. Gaffan, D. Olton, and S. Suomi (1988) The effects of fornix transection and of cingulate cortical ablation on spatial memory in rhesus monkeys. Exp. Brain Res. (in press).

O'Keefe, J. (1976) Place units in the hippocampus of the freely moving rat. Exp. Neurol. 51: 78-109.

O'Keefe, J. (1979) A review of the hippocampal place cells. Prog. Neurobiol. 13: 419-439.

O'Keefe, J., and J. Dostrovsky (1971) The hippocampus as a spatial map: Preliminary evidence from unit activity in the freely-moving rat. Brain Res. 34: 171-175.

O'Keefe, J., and L. Nadel (1978) The Hippocampus as a Cognitive Map, Clarendon, Oxford, UK.

O'Keefe, J., and L. Nadel (1979) Precis of O'Keefe and Nadel's The hippocampus as a cognitive map. Behav. Brain Sci. 2: 487-533.

O'Keefe, J., L. Nadel, S. Keightley, and D. Kill (1975) Fornix lesions selectively abolish place learning in the rat. Exp. Neurol. 48: 152166.

Olton, D. S., and R. J. Samuelson (1976) Remembrance of places passed. Spatial memory in rats. J. Exp. Psychol.: Anim. Behav. Processes 2: 97-116.

Pandya, D. N., G. W. Van Hoesen, and M. M. Mesulam (1981) Efferent connections of the cingulate gyrus in the rhesus monkey. Exp. Brain. Res. 42: 319-330.

Parkinson, J. K., and D. Medin (1983) Emerging attributes in monkey short-term memory. J. Exp. Psychol.: Anim. Behav. Processes 9: 3140.

Parkinson, J. K., and M. Mishkin (1982) A selective mnemonic role for the hippocampus in monkeys: Memory for the location of objects. Soc. Neurosci. Abstr. 8: 23.

Phillips, R. R., B. L. Malamut, and M. Mishkin (1983) Memory for stimulus-reward associations in the monkey is more severely affected by amygdalectomy than by hippocampectomy. Soc. Neurosci. Abstr. 9: 638 .

Pohl, W. (1973) Dissociation of spatial discrimination deficits following frontal and parietal lesions in monkeys. J. Comp. Physiol. Psychol. 82: 227-239.

Ranck, J. B., Jr. (1973) Studies on single neurons in dorsal hippocampal formation and septum in unrestrained rats: $I$. Behavioral correlates and firing repertoires. Exp. Neurol. 41: 461-531.

Saunders, R. C., E. A. Murray, and M. Mishkin (1984) Further evidence that amygdala and hippocampus contribute equally to visual recognition. Neuropsychologia 22: 785-796.

Seltzer, B., and G. W. Van Hoesen (1979) A direct inferior parietal lobule projection to the presubiculum in the rhesus monkey. Brain Res. 179: 157-161.

Smith, M., and B. Milner (1981) The role of the right hippocampus in the recall of spatial location. Neuropsychologia 19: 781-793.

Smith, M., and B. Milner (1983) Effects of focal brain lesions on sensitivity to frequency of occurrence. Soc. Neurosci. Abstr. 9: 30.

Smith, M., and B. Milner (1988) Right hippocampal impairment in location-recall: Encoding deficit or rapid forgetting? Neuropsychologia (in press)

Spiegler, B. J., and M. Mishkin (1981) Evidence for the sequential participation of inferior temporal cortex and amygdala in the acquisition of stimulus-reward associations. Behav. Brain Res. 3: 303-317.

Thomas, G. J., and P. S. Spafford (1984) Deficits for representational memory induced by septal and cortical lesions (singly and combined) in rats. Behav. Neurosci. 98: 394-404.

Waxler, M., and H. E. Rosvold (1970) Delayed alternation in monkeys after removal of the hippocampus. Neuropsychologia 8: 137-146.

Weiskrantz, L. (1956) Behavioral changes associated with ablation of the amygdaloid complex in monkeys. J. Comp. Physiol. Psychol. 49. 381-391.

Zola-Morgan, S., and L. R. Squire (1985) Medial temporal lesions in monkeys impair memory on a variety of tasks sensitive to human amnesia. Behav. Neurosci. 99: 22-34. 